\title{
Roles of IDH1/2 and TET2 mutations in myeloid disorders
}

\author{
Satoshi Inoue $^{1} \cdot$ François Lemonnier $^{1} \cdot$ Tak W. Mak $^{1}$
}

Received: 1 February 2016 / Revised: 22 February 2016 / Accepted: 2 March 2016 / Published online: 15 March 2016

(C) The Japanese Society of Hematology 2016

\begin{abstract}
Mutations of the epigenetic enzymes isocitrate dehydrogenase (IDH) 1 and 2, and the methylcytosine dioxygenase 'ten-eleven translocation 2' (TET2), are common in human myeloid malignancies and drivers of these disorders but the underlying mechanisms remain obscure. This review examines mutant IDH1/2 and TET2 enzymes in the context of responses to DNA damage and their potential involvement in age-related genomic instability. The clinical relevance of these findings and their potential application in novel therapeutic strategies is also discussed.
\end{abstract}

Keywords IDH1 $\cdot$ TET2 2 MDS $\cdot$ AML $\cdot$ DNA damage · DNA repair

\section{Introduction}

Recent advances in genomic sequencing have unveiled numerous novel somatic gene mutations in cancer cells, enabling characterization of unexpected functions for such genes. In myeloid malignancies such as myelodysplastic syndrome (MDS) and acute myeloid leukemia (AML), mutations in genes involved in epigenetics, including isocitrate dehydrogenases (IDH) 1 and 2, and methylcytosine dioxygenase 'ten-eleven translocation 2' (TET2), are not only common but also drivers of these diseases.

S. Inoue and F. Lemonnier contributed equally to this work.

Tak W. Mak

tmak@uhnresearch.ca

1 The Campbell Family Institute for Breast Cancer Research, Princess Margaret Cancer Centre, Ontario Cancer Institute, University Health Network, 610 University Avenue, Toronto, ON M5G 2M9, Canada
Although mutated IDH1/2 are clearly established as oncogenes in myeloid cells, the mechanisms underlying their tumorigenic effects are not clear. Much evidence suggests that mutant IDH enzymes exert their effects via inhibition of TET2, but important clinical differences between IDH1-mutant and TET2-mutant hematopoietic disorders suggest that the oncogenic mechanisms of these mutated enzymes may differ. In particular, divergent effects of mutant IDH1/2 and TET2 on DNA damage repair mechanisms have been identified. In this review, we examine mutant IDH1/2 and TET2 in the context of responses to DNA damage and their potential involvement in genomic instability. We also discuss the clinical relevance of these findings and their potential application in novel therapeutic strategies.

\section{Mutation of TET family enzymes}

The TET family of dioxygenases is highly conserved and contains three members: TET1, -2 and -3 . Somatic mutations of TET2 occur in various myeloid disorders, including chronic myelomonocytic leukemia (CMML) ( $50 \%)$, myeloid proliferative neoplasm (MPN) ( 13\%), MDS $(\sim 25 \%)$ and AML $(\sim 23 \%)$ [1, 2]. TET2 is also mutated in $\mathrm{B}$ and $\mathrm{T}$ cell lymphoid malignancies, including angioimmunoblastic T cell lymphoma (AITL) [3]. More recently, TET2 mutations have been detected in elderly individuals with clonal hematopoiesis of indeterminate potential, i.e.: individuals with clonal hematopoiesis without identified hematological disease. This indicates that TET2 mutations alone cannot drive leukemogenesis and that additional events probably contribute $[4,5]$. Interestingly, TETI and TET3 mutations are rare in human hematological diseases [6]. This predominance of TET2 alterations is not 
understood, particularly as conditional knockout (KO) of TET 1 , TET $1 / 2$ or TET $2 / 3$ in murine hematopoietic cells has profound biological consequences [7-9].

At the molecular level, TET enzymes catalyze the $\mathrm{Fe}(\mathrm{II})$ - and $\alpha$-ketoglutarate $(\alpha \mathrm{KG})$-dependent hydroxylation of 5-methylcytosine $(5 \mathrm{mC})$ residues in DNA to form 5 -hydroxymethylcytosine $(5 \mathrm{hmC})$, which is involved in epigenetic regulation of gene expression [10]. $5 \mathrm{hmC}$ and other modifications trigger various mechanisms of DNA demethylation [11], and 5hmC inhibits the recruitment of methyl-DNA-binding transcriptional repressors to gene promoters. TET proteins also interact with O-linked $\beta-N$ acetylglucosamine (O-GlcNAc) transferase (OGT), which is responsible for the O-GlcNAcylation of histones that regulates gene transcription [12].

TET activity is essential for normal development in vivo. $5 \mathrm{hmC}$ is enriched in wild type (WT) murine embryonic stem (ES) cells, such that TET1/2/3 KO ES cells are dramatically depleted of $5 \mathrm{hmC}$ and exhibit poorly differentiated embryonic bodies and teratomas [13]. Promoter DNA in these embryos is hypermethylated, and genes involved in embryonic development and differentiation are deregulated. Similarly, in murine hematopoietic stem cells (HSCs) and progenitors, TET2 deficiency leads to impaired differentiation and increased self-renewal potential that drives a disease mimicking human CMML [14, 15]. However, as is true in humans, the development of AML in TET2deficient mice requires additional alterations, such as a gain-of-function mutation of FLT3 [16]. Whether TET1/3 can compensate for loss of TET2 has yet to be addressed, but no increase in TET1/3 expression has been detected in TET2-deficient mice [14]. Moreover, human TET2-mutated AML cells show a decrease in $5 \mathrm{hmC}$ levels [17]. These data indicate that the mutations ultimately responsible for TET2-mutated diseases abrogate TET enzymatic functions, and also suggest that the epigenetic alterations resulting from TET abrogation may be involved in tumorigenic transformation.

\section{Mutation of IDH1/2}

IDH1/2 are homodimeric $\mathrm{NADP}^{+}$-dependent enzymes that catalyze oxidative decarboxylation of isocitrate to produce the $\alpha \mathrm{KG}, \mathrm{NADP}$, and $\mathrm{CO}_{2}$ required for the Krebs cycle. In WT cells, IDH1 localizes to the cytoplasm and peroxisomes, whereas IDH2 resides in the mitochondrial matrix [18]. More than 80 dioxygenases require IDH-derived $\alpha \mathrm{KG}$ to complete their enzymatic reactions. In particular, $\alpha \mathrm{KG}$ regulates TET DNA hydroxylases and Jumonji C (JmjC) domain-containing histone demethylases (Fig. 1). $\alpha \mathrm{KG}$ is abundant in ES cells and required for their pluripotent activity.
In 2008, sequencing of human tumor genomic DNA revealed missense mutations of IDH1/2 in glioma ( 90\%), chondrosarcoma ( 50\%), cholangiosarcoma (10-23\%), MPN (2.5 \%), MDS (3.5\%) and AML (15-33\%) [1, 2, 18]. These mutations are heterozygous, mutually exclusive, and usually occur at one of three specific arginine residues: IDH1-R132, IDH2-R140 or IDH2-R172. Mutation of IDH2-R140 occurs exclusively in hematological disorders (usually myeloid) and not in solid tumors, whereas IDH2R172 mutation is common in AITL [19].

Metabolite profiling studies later revealed that mutant IDH enzymes acquire a neomorphic activity that converts $\alpha \mathrm{KG}$ to D-2-hydroxyglutarate (D2HG) in a reaction consuming NADPH [20]. This abnormal reaction is due to increased affinity of mutated IDH1/2 for $\alpha \mathrm{KG}$ and decreased affinity for isocitrate. D2HG is an enigmatic metabolite in WT cells because it does not appear to function in any known metabolic process but is nevertheless detectable at low levels in both its D- and L-enantiometric forms. Both isoforms can be recycled back to $\alpha \mathrm{KG}$ by D-2-hydroxyglutarate dehydrogenase (D2HGDH) [21] and L-2-hydroxyglutarate dehydrogenase (L2HGDH) [22]. D2HG was first identified in patients with D-2-hydroxyaciduria, which is caused by alterations to the D2HGDH gene or by IDH2-R140 mutations [23]. Levels of L2HG, but not D2HG, increase in cell cultured under hypoxia, and this elevation is associated with increased trimethyl H3K9. Trimethyl H3K9 is a marker of transcriptional repression, suggesting the involvement of L2HG in cell adaptation to hypoxia [24, 25]. However, the biological importance of the differing phenotypes caused by $\mathrm{D} 2 \mathrm{HG}$ and $\mathrm{L} 2 \mathrm{HG}$ has yet to be confirmed in vivo.

The structural similarity between $\alpha \mathrm{KG}$ and $\mathrm{D} 2 \mathrm{HG}$ has led to the hypothesis that $\mathrm{D} 2 \mathrm{HG}$ is a competitive inhibitor of $\alpha$ KG-dependent reactions. 2 2HG has a greater capacity than D2HG to inhibit dioxygenases [26] but, to date, L2HG has not been implicated in oncogenesis, unlike D2HG. Dioxygenases vary in their vulnerability to inhibition by $\mathrm{D} 2 \mathrm{HG}$, ranging from the micromolar level for histone demethylases to the millimolar level for prolyl hydroxylase [26]. With respect to epigenetic enzymes, mutant IDH1/2 block TET1/2/3, resulting in decreased $5 \mathrm{hmC}$ and a hypermethylator phenotype in gliomas [27]. Excessive D2HG also inhibits JmjC histone demethylases, increasing levels of trimethylation on various histones. However, the physiological consequences of these changes to histone marking are unclear.

D2HG-mediated inhibition is not restricted to epigenetic enzymes. We found, using a conditional IDH1 R132 knockin (KI) mouse model, that D2HG impairs collagen maturation and may influence HIF1 $\alpha$ and responses to hypoxia in brain [28]. In vitro, $\mathrm{Xu}$ et al. demonstrated that $\mathrm{D} 2 \mathrm{HG}$ can inhibit HIF $1 \alpha$ degradation by blocking proline hydroxylation [29], but Koivunen et al. then showed that D2HG can also activate prolyl hydroxylase domain-containing protein 2 , 


\section{IDH1/2 WT}

IDH1/2 mutant

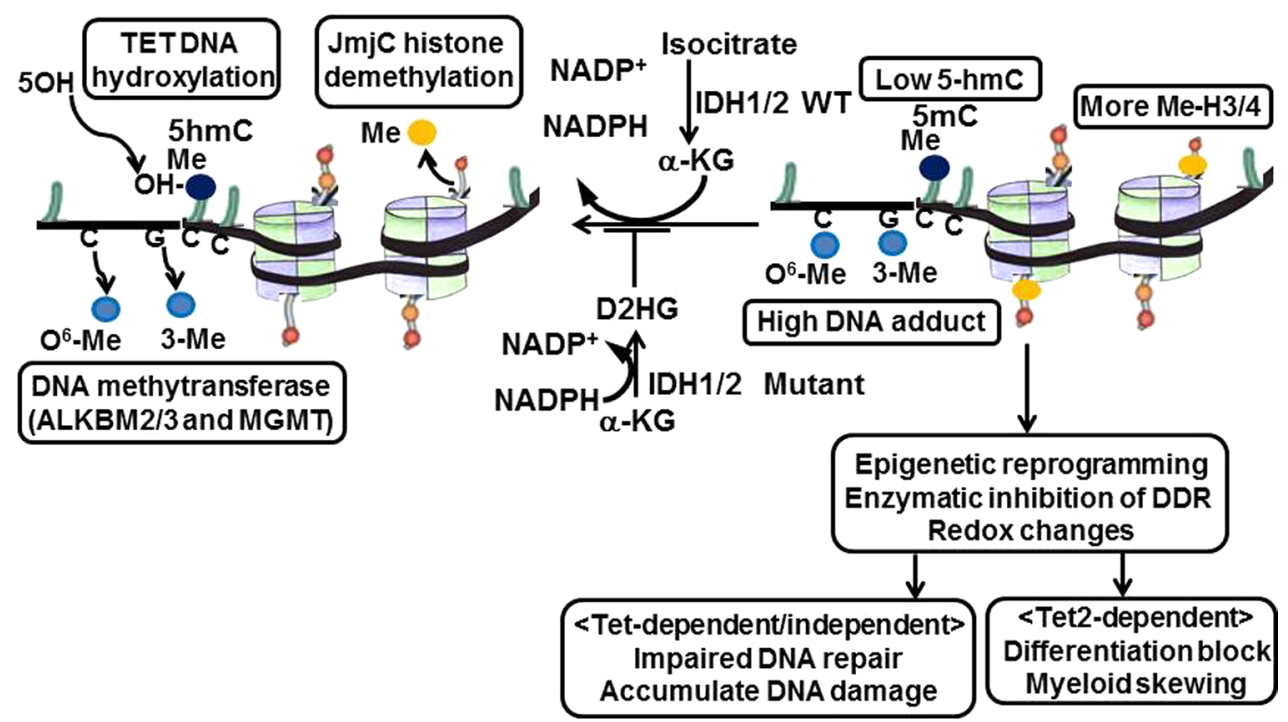

Fig. 1 Model of the effects of IDH1/2 mutations on DDR/DNA repair. The wild type IDH $1 / 2$ enzymes produce $\alpha$-ketoglutarate $(\alpha-K G)$ and NAPDH. $\alpha-K G$ is required for the function of many enzymes, including DNA hydroxymethylases (TET family), histone demethylases (JmjC family) and DNA methyltransferases (ALKBH2/3 and MGMT). TET enzymes hydroxylate 5-methylcytosine $(5 \mathrm{mC})$ to produce 5-hydroxymethylcytosine $(5 \mathrm{hmC})$, while $\mathrm{JmjC}$ enzymes remove methyl groups (Me) from the lysine residues of histones H3/4. These reactions regulate the histone code and chromatin structure. If a single-strand DNA break (SSB) naturally occurs, the DNA repair enzymes ALKBH2/3 and MGMT act to maintain genomic integrity. MGMT converts mutagenic $O-6$-methylguanine to guanine whereas ALKBH2/3 remove the methyl groups from muta-

which is encoded by Egln1 and decreases HIF1 $\alpha$ levels [30]. In vivo, IDH1-R132-KI embryos show heightened HIF1 $\alpha$ in the brain but no changes in the hematopoietic system [28, 31 ], suggesting that effects of $\mathrm{D} 2 \mathrm{HG}$ on HIF1 $\alpha$ are likely cell context-dependent. Lastly, in AML cell lines overexpressing IDH1-R132H, D2HG inhibited Cox IV and mitochondrial respiration in a manner dependent on $\mathrm{Bcl} 2$ [32].

IDH1/2 mutations may also have consequences that are independent of D2HG. In contrast to WT IDH1/2, which produce $\mathrm{NADPH}$, mutated IDH1/2 produce $\mathrm{NADP}^{+}$, which drives an increase in reactive oxygen species (ROS) that may alter hematopoietic differentiation or promote tumor transformation. In addition, because WT IDH1/2 activities are crucial for metabolism, IDH1/2 mutations disrupt normal Krebs cycle functioning and may alter the synthesis of amino acids and fatty acids [18].

\section{TET2 and IDH1/2 mutations in hematological disorders}

IDH1/2 and TET2 mutations affect epigenetic regulation and tend to be early events in leukemogenesis [33, genic 1-methyladenine and 3-methylcytosine and convert them to adenine and cytosine. All these $\alpha-K G$-dependent reactions maintain the appropriate status of epigenetics and DNA repair in hematopoietic stem cells (HSCs). On the other hand, mutant IDH1/2 convert $\mathrm{NADPH}$ and $\alpha-\mathrm{KG}$ and to $\mathrm{NADP}^{+}$and D-hydroxyglutarate (D2HG). Because D2HG competitively inhibits $\alpha$-KG-dependent reactions, mutant IDH1/2 enzymes heighten DNA damage (particularly DNA adducts) by not only disrupting epigenetic regulators involved in DDR/DNA repair but also by elevating oxidative stress due to increased $\mathrm{NADP}^{+}$production. The combined effects of all these changes lead to a block in myeloid differentiation and accumulation of DNA damage in $\mathrm{HSC} /$ myeloid progenitors that is oncogenic

34]. TET2- or IDH-mutated hematopoietic cells display an overlapping DNA hypermethylation signature [35] that is associated with decreased $5 \mathrm{hmC}$ [17]. While IDH1/2 mutations are linked to lower levels of 5hmC than TET2 mutations, this difference is explained by the fact that some TET2-mutated patients are haploinsufficient for this enzyme. In MDS and AML patients, the driver $I D H 1 / 2$ and TET2 mutations coexist with similar "secondary" mutations, including alterations affecting FLT3, NPMI and RAS [33, 35-37]. Likewise, TET2 KO mice and IDH1-R132-KI mice show parallel phenotypes, including global $5 \mathrm{hmC}$ reduction, altered DNA methylation, blocked HSC/progenitor differentiation, myeloid skewing, and myeloid disorders $[14,15,31]$.

Despite the above, substantial differences exist between IDH1/2-mutated and TET2-mutated hematological disorders. TET2 mutation is more common in MDS/MPN than in AML, whereas $I D H 1 / 2$ mutations are more frequent in AML than MDS/MPN $[1,2]$. When present in MDS, IDHI mutation is associated with shorter leukemia-free survival and decreased overall survival, whereas these impacts are not found in TET2-mutated MDS [38, 39]. Furthermore, 
unlike TET2 mutations, IDH1/2 mutations are quite rare in clonal hematopoiesis of indeterminate potential. IDHI/2 mutation frequency is higher in de novo AML than in chronic myeloid neoplasms [40], whereas the greatest incidence $(50 \%)$ of TET2 mutations occurs in CMML, in agreement with the myelomonocytic proliferation in TET2 KO mice. Thus, IDH1/2 mutations appear to have higher oncogenic potential than TET2 mutations and are associated with more aggressive diseases $[1,2]$.

A key observation has been that IDHI/2 and TET2 mutations are mutually exclusive in myeloid neoplasms. Since D2HG inhibits TET2 enzymatic activity, it has been postulated that the effects of mutated IDH in MDS/AML are due solely to D2HG-mediated TET2 inhibition and the resulting alterations to DNA methylation in HSCs and myeloid progenitors [18]. This hypothesis is in line with the strong inhibition of TET2 activity in cells of patients with IDHmutated AML [35]. However, AITL, which is one of the most frequent $\mathrm{T}$ cell lymphomas, stands in contrast to the preceding. These lymphomas often exhibit TET2 mutations $(50 \%)$ or $I D H 2$ mutations (20-30\%). Surprisingly, these two mutations are not mutually exclusive and coexist in the same tumor in 60-100\% of IDH2-mutated AITL patients $[41,42]$. Thus, TET2 mutation and IDHI/2 mutation have at least some differing tumorigenic effects, even if they share a communal oncogenic mechanism.

The prognostic impact of IDH1/2 mutations in AML is unclear, and could depend on the specific IDH mutational hotspot and on associated secondary mutations. However, in some studies, AML patients with both $I D H 1 / 2$ mutation and NPM1 mutation have a better prognosis after treatment with the daunorubicin than do TET2-mutated AML patients treated with this drug [43]. As daunorubicin is a DNA damage-targeting drug, this result could be explained by an effect of mutated IDH1/2 on the repair of damaged DNA. These findings are of great interest, and investigation of this possibility in multiple and larger patient cohorts would help to clarify this point.

\section{Repair of damaged DNA in HSCs}

HSCs continuously self-renew throughout life, whereas hematopoietic progenitors are relatively short-lived and differentiate into mature blood cells. It is widely believed that HSCs must possess unique cyto- and geno-protective mechanisms to ensure their homeostasis and long-term functionality. Indeed, WT murine HSCs are extremely quiescent, maintaining low levels of glycolytic metabolism and ROS. This quiescence is an attempt to minimize the chance of introducing genomic errors that could be caused by endogenous stressors such as cellular respiration and DNA replication.
In response to DNA damage, normal cells activate the DNA damage response (DDR), which is mediated by intracellular cell signaling that implement cell-cycle checkpoint controls and repair DNA breaks. A cell first activates the sensor kinases, such as ATM and ATR, which recognize DNA lesions and recruit the appropriate effectors to either arrest the cell and repair the damage or induce apoptosis. For double-strand DNA breaks (DSBs), two major DNA repair pathways are utilized: non-homologous end-joining (NHEJ) and homology-directed repair (HDR). There is a plethora of proteins, including ATM, Chk2 and p53, that play critical roles in these processes. Most single-strand breaks (SSBs) are dealt with by base excision repair (BER), nucleotide excision repair (NER), or DNA mismatch repair (MMR). Defects in many of these elements are associated with tumorigenesis [44].

HSCs are unique in that most DNA damage and repair signals are attenuated in these cells [45], leaving active only the rapid but error-prone NHEJ mechanism [46]. Next generation whole-genome sequencing of HSC cDNAs from normal humans of various ages has confirmed that HSCs serve as a reservoir for the accumulated DNA damage underlying hematopoietic disorders such as AML and MDS [33, 47]. Initial mutations of driver genes, including IDH1/2 and TET2, that occur in HSCs can clonally progress as preleukemic events, eventually leading to AML $[1,48]$.

In mice, HSCs lacking components of DDR and DNA repair pathways have significant deficits. The $\gamma \mathrm{H} 2 \mathrm{AX}$ foci that indicate DNA damage and accumulate in HSCs of older WT mice are found in even greater numbers in HSCs of mice lacking DDR function [49-51]. Mutations in DNA damage and repair genes result in unrepaired DNA damage in murine HSCs that leads to accelerated decline of their self-renewal capacity with age.

In contrast to most of the DNA repair mechanisms described above, there are two types of methyltransferases, namely members of the Alkylation Repair Homolog (ALKBH) family and $O$-6-methylguanine-DNA methyltransferase (MGMT), that can repair alkylated DNA using chemical reversion processes that do not require a nucleotide template. ALKBH enzymes convert the mutagenic nucleotides 1-methyladenine and 3-methylcytosine to adenine and cytosine, respectively [44]. ALKBH enzymatic activity depends on $\alpha \mathrm{KG}$ and so may be inhibited by the $\mathrm{D} 2 \mathrm{HG}$ produced by mutant IDH1/2, allowing alkylated DNA damage to accumulate. Another example is the conversion of the naturally-occurring, mutagenic nucleotide $O$-6-methylguanine to guanine by MGMT [44]. Notably, the MGMT gene is epigenetically silenced in several cancers [6]. Temozolomide is an alkylating agent that converts guanine to $O-6$-methylguanine, an event that eventually precipitates the death of the tumor cells. Indeed, temozolomide treatment of AML patients whose tumor cells 
show MGMT downregulation has shown clinical promise [52]. Thus, it is interesting to investigate levels of MGMT expression in IDHI/2 and TET2 mutated AML.

Since 2015, the roles of the IDH and TET family enzymes in DDR/DNA repair have been investigated. TET1 KO mice develop spontaneous B cell lymphomas, and gene set enrichment analysis of RNA-seq from TET1 KO LSK and MPP cells showed that DNA repair genes, particularly the BER genes Apex1, Lig1 and Exo1, were downregulated [7]. Accordingly, $\gamma \mathrm{H} 2 \mathrm{AX}$ foci were prominent in TET1 $\mathrm{KO}$ pro-B cells. Intriguingly, whole-exome sequencing of human TET1-deficient non-Hodgkin B cell lymphomas revealed an increase in missense mutations, suggesting that TET1 loss can heighten the mutational burden in this lymphoma, even it does not lead to myeloid malignancy [7].

In contrast to TET $1 \mathrm{KO}$ mice, TET $2 / 3$ DKO mice develop AML within 2 months [8]. Gene expression analysis demonstrated deregulation of HR- and NHEJ-related genes in GMP and CD11 $b^{+}$myeloid cells of these mutants but a much milder effect in LSK cells. Although TET3 mutation is not clinically relevant in humans, these mouse studies emphasize that a decrease in $5 \mathrm{hmC}$ levels impinges in multiple ways on the repair of damaged DNA. Interestingly, there is so far no evidence of DDR alterations in TET2 KO mice or TET2 mutated AML patients. It may be that TET2 alterations alone are unable to impair DDR, and that additional anomalies, such as TET3 or TET1 mutations, are required to derail this response.

An effect of IDH1 mutation on DNA damage/repair was also demonstrated by Molenaar et al. using an HCT116 cell line expressing mutant IDH1-R132 [53]. In this system, decreased NADPH production due to the mutated IDH1 enzyme increased ROS, leading to DNA damage induced by oxidative stress (Fig. 1). Indeed, HCT116 cells expressing mutant IDH1-R132 showed increased radio-sensitivity that was reversed by AGI-5198, a specific inhibitor of mutant IDH1 enzymes [54]. Also, Wang et al. used U87MG and HT1080 cell lines overexpressing mutant IDH1 to show that D2HG inhibits the ALKBH family of DNA repair enzymes [55]. As ALKBH activities depend on $\alpha \mathrm{KG}$, these cell lines showed decreased DNA repair, accumulation of DNA damage, and sensitivity to alkylating agents such as methyl methanesulfonate (MMS) and $N$-methyl- $N^{\prime}$-nitro- $N$-nitroguanidine (MNNG) (Fig. 1). Interestingly, although the above reports indicate that mutant IDH1R132 reduces DNA repair capacity, their underlying mechanisms differ. It is possible that these differences, which remain under investigation, are cell context-dependent.

\section{Clinical implications}

The clinical relevance of defects in DDR/DNA repair in AML has been controversial because AML has a low mutational burden and key DDR/DNA repair genes are rarely mutated in this disease [6]. It may be relevant that AML arises primarily de novo and is characterized by a block in myeloid progenitor differentiation that leads to abnormal expansion. Slush et al. reported that clonal AML arises from a pool of preleukemic cells representing a heterogeneous group of subpopulations [36, 37]. Genomic diversity was observed only in non-clonally expanded populations and not in clonally expanded homogeneous populations. Thus, the bulk DNA sequencing strategy commonly used for TCGA analysis could be inappropriate in the AML context because the detection limit is too low to identify a non-clonal mutation even if it exists. The deep sequencing of genomic DNA isolated from non-clonally expanded single cells from patients with TET2- or IDH1/2-mutated AML should provide insight into the true effects of these mutations on genomic instability in AML.

The findings from mouse models that mutant IDH and TET enzymes do influence DDR/DNA repair and thus potentially transformation point towards new therapeutic strategies for IDH-mutated cancers. Several drugs that target DDR by exerting genotoxic stress have a long history of clinical use, including for AML. These agents kill tumor cells by alkylating DNA [carmustine (BCNU) and temozolomide], crosslinking DNA (cisplatin), or inhibiting DNA replication (gemcitabine, daunorubicin, fludarabine). Other drugs, currently in clinical trials, target specific DDR molecules, such as PARP (Olaparib), ATR (AZD6738), and MGMT (Lomeguatrib). A combination approach may also be of benefit. In preclinical models of AML, PARP inhibitors are most effective in killing AML cells in which the HDR repair mechanism is also impaired [56]. Thus, it will be interesting to investigate the sensitivity of IDH1/2- and TET2-mutant AML to PARP inhibitors. Other genes may also affect the efficacy of DDR targeting agents, and, due to the complex relationship between DDR and hematopoietic differentiation, mutant IDH1-expressing cells may harbor other targetable vulnerabilities. All these possibilities require investigation in large and independent patient cohorts.

\section{Closing remarks}

This review has examined mutant IDH1/2 and TET2 enzymes in the context of DDR and their potential involvement in tumorigenic genomic instability. The clinical relevance of these findings is becoming clear, and exciting new therapeutic strategies exploiting these observations should improve treatment options for patients with hematological disorders. 


\section{References}

1. Shih AH, Abdel-Wahab O, Patel JP, Levine RL. The role of mutations in epigenetic regulators in myeloid malignancies. Nat Rev Cancer. 2012;12(9):599-612.

2. Xie M, Lu C, Wang J, McLellan MD, Johnson KJ, Wendl MC, et al. Age-related mutations associated with clonal hematopoietic expansion and malignancies. Nat Med. 2014;20(12):1472-8.

3. Lemonnier F, Couronne L, Parrens M, Jais JP, Travert M, Lamant $\mathrm{L}$, et al. Recurrent TET2 mutations in peripheral T-cell lymphomas correlate with TFH-like features and adverse clinical parameters. Blood. 2012;120(7):1466-9.

4. Jaiswal S, Fontanillas P, Flannick J, Manning A, Grauman PV, Mar BG, et al. Age-related clonal hematopoiesis associated with adverse outcomes. N Engl J Med. 2014;371(26):2488-98.

5. Busque L, Patel JP, Figueroa ME, Vasanthakumar A, Provost S, Hamilou Z, et al. Recurrent somatic TET2 mutations in normal elderly individuals with clonal hematopoiesis. Nat Genet. 2012;44(11):1179-81.

6. Cancer Genome Atlas Research Network. Genomic and epigenomic landscapes of adult de novo acute myeloid leukemia. N Engl J Med. 2013;368(22):2059-74.

7. Cimmino L, Dawlaty MM, Ndiaye-Lobry D, Yap YS, Bakogianni S, Yu Y, et al. TET1 is a tumor suppressor of hematopoietic malignancy. Nat Immunol. 2015;16(6):653-62.

8. An J, Gonzalez-Avalos E, Chawla A, Jeong M, Lopez-Moyado IF, Li W, et al. Acute loss of TET function results in aggressive myeloid cancer in mice. Nat Commun. 2015;6:10071.

9. Zhao Z, Chen L, Dawlaty MM, Pan F, Weeks O, Zhou Y, et al. Combined loss of Tet 1 and Tet 2 promotes B cell, but not myeloid malignancies, in mice. Cell Rep. 2015;13(8):1692-704.

10. Ito S, Shen L, Dai Q, Wu SC, Collins LB, Swenberg JA, et al. Tet proteins can convert 5-methylcytosine to 5-formylcytosine and 5-carboxylcytosine. Science. 2011;333(6047):1300-3.

11. Guo JU, Su Y, Zhong C, Ming GL, Song H. Hydroxylation of 5-methylcytosine by TET1 promotes active DNA demethylation in the adult brain. Cell. 2011;145(3):423-34.

12. Chen Q, Chen Y, Bian C, Fujiki R, Yu X. TET2 promotes histone O-GlcNAcylation during gene transcription. Nature. 2013;493(7433):561-4.

13. Dawlaty MM, Breiling A, Le T, Barrasa MI, Raddatz G, Gao Q, et al. Loss of Tet enzymes compromises proper differentiation of embryonic stem cells. Dev Cell. 2014;29(1):102-11.

14. Moran-Crusio K, Reavie L, Shih A, Abdel-Wahab O, NdiayeLobry D, Lobry C, et al. Tet 2 loss leads to increased hematopoietic stem cell self-renewal and myeloid transformation. Cancer Cell. 2011;20(1):11-24.

15. Quivoron C, Couronne L, Della Valle V, Lopez CK, Plo I, Wagner-Ballon $\mathrm{O}$, et al. TET2 inactivation results in pleiotropic hematopoietic abnormalities in mouse and is a recurrent event during human lymphomagenesis. Cancer Cell. 2011;20(1):25-38.

16. Shih AH, Jiang Y, Meydan C, Shank K, Pandey S, Barreyro L, et al. Mutational cooperativity linked to combinatorial epigenetic gain of function in acute myeloid leukemia. Cancer Cell. 2015;27(4):502-15.

17. Kroeze LI, Aslanyan MG, van Rooij A, Koorenhof-Scheele TN, Massop M, Carell T, et al. Characterization of acute myeloid leukemia based on levels of global hydroxymethylation. Blood. 2014;124(7):1110-8.

18. Cairns RA, Mak TW. Oncogenic isocitrate dehydrogenase mutations: mechanisms, models, and clinical opportunities. Cancer Discov. 2013;3(7):730-41.

19. Cairns RA, Iqbal J, Lemonnier F, Kucuk C, de Leval L, Jais JP, et al. IDH2 mutations are frequent in angioimmunoblastic T-cell lymphoma. Blood. 2012;119(8):1901-3.
20. Dang L, White DW, Gross S, Bennett BD, Bittinger MA, Driggers EM, et al. Cancer-associated IDH1 mutations produce 2-hydroxyglutarate. Nature. 2009;462(7274):739-44.

21. Achouri Y, Noel G, Vertommen D, Rider MH, Veiga-Da-Cunha $M$, Van Schaftingen E. Identification of a dehydrogenase acting on D-2-hydroxyglutarate. Biochem J. 2004;381(Pt 1):35-42.

22. Rzem R, Veiga-da-Cunha $M$, Noel $G$, Goffette S, Nassogne MC, Tabarki B, et al. A gene encoding a putative FADdependent L-2-hydroxyglutarate dehydrogenase is mutated in L-2-hydroxyglutaric aciduria. Proc Natl Acad Sci USA. 2004;101(48):16849-54.

23. Kranendijk M, Struys EA, van Schaftingen E, Gibson KM, Kanhai WA, van der Knaap MS, et al. IDH2 mutations in patients with D-2-hydroxyglutaric aciduria. Science. 2010;330(6002):336.

24. Intlekofer AM, Dematteo RG, Venneti S, Finley LW, Lu C, Judkins AR, et al. Hypoxia induces production of L-2-hydroxyglutarate. Cell Metab. 2015;22(2):304-11.

25. Oldham WM, Clish CB, Yang Y, Loscalzo J. Hypoxia-mediated increases in L-2-hydroxyglutarate coordinate the metabolic response to reductive stress. Cell Metab. 2015;22(2):291-303.

26. Chowdhury R, Yeoh KK, Tian YM, Hillringhaus L, Bagg EA, Rose NR, et al. The oncometabolite 2-hydroxyglutarate inhibits histone lysine demethylases. EMBO Rep. 2011;12(5):463-9.

27. Turcan S, Rohle D, Goenka A, Walsh LA, Fang F, Yilmaz E, et al. IDH1 mutation is sufficient to establish the glioma hypermethylator phenotype. Nature. 2012;483(7390):479-83.

28. Sasaki M, Knobbe CB, Itsumi M, Elia AJ, Harris IS, Chio II, et al. D-2-hydroxyglutarate produced by mutant IDH1 perturbs collagen maturation and basement membrane function. Genes Dev. 2012;26(18):2038-49.

29. Xu W, Yang H, Liu Y, Yang Y, Wang P, Kim SH, et al. Oncometabolite 2-hydroxyglutarate is a competitive inhibitor of alpha-ketoglutarate-dependent dioxygenases. Cancer Cell. 2011;19(1):17-30.

30. Koivunen P, Lee S, Duncan CG, Lopez G, Lu G, Ramkissoon S, et al. Transformation by the $(R)$-enantiomer of 2-hydroxyglutarate linked to EGLN activation. Nature. 2012;483(7390):484-8.

31. Sasaki M, Knobbe CB, Munger JC, Lind EF, Brenner D, Brustle A, et al. IDH1 (R132H) mutation increases murine haematopoietic progenitors and alters epigenetics. Nature. 2012;488(7413):656-9.

32. Chan SM, Thomas D, Corces-Zimmerman MR, Xavy S, Rastogi $\mathrm{S}$, Hong WJ, et al. Isocitrate dehydrogenase 1 and 2 mutations induce BCL-2 dependence in acute myeloid leukemia. Nat Med. 2015;21(2):178-84.

33. Welch JS, Ley TJ, Link DC, Miller CA, Larson DE, Koboldt DC, et al. The origin and evolution of mutations in acute myeloid leukemia. Cell. 2012;150(2):264-78.

34. Delhommeau F, Dupont S, Della Valle V, James C, Trannoy S, Masse A, et al. Mutation in TET2 in myeloid cancers. N Engl J Med. 2009;360(22):2289-301.

35. Figueroa ME, Abdel-Wahab O, Lu C, Ward PS, Patel J, Shih A, et al. Leukemic IDH1 and IDH2 mutations result in a hypermethylation phenotype, disrupt TET2 function, and impair hematopoietic differentiation. Cancer Cell. 2010;18(6):553-67.

36. Corces-Zimmerman MR, Hong WJ, Weissman IL, Medeiros BC, Majeti R. Preleukemic mutations in human acute myeloid leukemia affect epigenetic regulators and persist in remission. Proc Natl Acad Sci USA. 2014;111(7):2548-53.

37. Shlush LI, Zandi S, Mitchell A, Chen WC, Brandwein JM, Gupta V, et al. Identification of pre-leukaemic haematopoietic stem cells in acute leukaemia. Nature. 2014;506(7488):328-33.

38. Patnaik MM, Hanson CA, Hodnefield JM, Lasho TL, Finke CM, Knudson RA, et al. Differential prognostic effect of IDH1 versus IDH2 mutations in myelodysplastic syndromes: a Mayo Clinic study of 277 patients. Leukemia. 2012;26(1):101-5. 
39. Thol F, Weissinger EM, Krauter J, Wagner K, Damm F, Wichmann M, et al. IDH1 mutations in patients with myelodysplastic syndromes are associated with an unfavorable prognosis. Haematologica. 2010;95(10):1668-74.

40. Molenaar RJ, Thota S, Nagata Y, Patel B, Clemente M, Przychodzen B, et al. Clinical and biological implications of ancestral and non-ancestral IDH1 and IDH2 mutations in myeloid neoplasms. Leukemia. 2015;29(11):2134-42.

41. Sakata-Yanagimoto M, Enami T, Yoshida K, Shiraishi Y, Ishii R, Miyake Y, et al. Somatic RHOA mutation in angioimmunoblastic T cell lymphoma. Nat Genet. 2014;46(2):171-5.

42. Wang C, McKeithan TW, Gong Q, Zhang W, Bouska A, Rosenwald $\mathrm{A}$, et al. IDH2R172 mutations define a unique subgroup of patients with angioimmunoblastic T-cell lymphoma. Blood. 2015;126(15):1741-52.

43. Patel JP, Gonen M, Figueroa ME, Fernandez H, Sun Z, Racevskis J, et al. Prognostic relevance of integrated genetic profiling in acute myeloid leukemia. N Engl J Med. 2012;366(12):1079-89.

44. Helleday T, Petermann E, Lundin C, Hodgson B, Sharma RA. DNA repair pathways as targets for cancer therapy. Nat Rev Cancer. 2008;8(3):193-204

45. Beerman I, Seita J, Inlay MA, Weissman IL, Rossi DJ. Quiescent hematopoietic stem cells accumulate DNA damage during aging that is repaired upon entry into cell cycle. Cell Stem Cell. 2014;15(1):37-50.

46. Mohrin M, Bourke E, Alexander D, Warr MR, Barry-Holson K, Le Beau MM, et al. Hematopoietic stem cell quiescence promotes error-prone DNA repair and mutagenesis. Cell Stem Cell. 2010;7(2):174-85.

47. Krivtsov AV, Figueroa ME, Sinha AU, Stubbs MC, Feng Z, Valk PJ, et al. Cell of origin determines clinically relevant subtypes of MLL-rearranged AML. Leukemia. 2013;27(4):852-60.

48. Jan M, Snyder TM, Corces-Zimmerman MR, Vyas P, Weissman IL, Quake SR, et al. Clonal evolution of preleukemic hematopoietic stem cells precedes human acute myeloid leukemia. Sci Transl Med. 2012;4(149):149ra18.
49. Ito K, Hirao A, Arai F, Matsuoka S, Takubo K, Hamaguchi I, et al. Regulation of oxidative stress by ATM is required for self-renewal of haematopoietic stem cells. Nature. 2004;431(7011):997-1002.

50. Nijnik A, Woodbine L, Marchetti C, Dawson S, Lambe T, Liu C, et al. DNA repair is limiting for haematopoietic stem cells during ageing. Nature. 2007;447(7145):686-90.

51. Rossi DJ, Bryder D, Seita J, Nussenzweig A, Hoeijmakers J, Weissman IL. Deficiencies in DNA damage repair limit the function of haematopoietic stem cells with age. Nature. 2007;447(7145):725-9.

52. Brandwein JM, Kassis J, Leber B, Hogge D, Howson-Jan K, Minden MD, et al. Phase II study of targeted therapy with temozolomide in acute myeloid leukaemia and high-risk myelodysplastic syndrome patients pre-screened for low $O(6)$-methylguanine DNA methyltransferase expression. $\mathrm{Br} \mathrm{J}$ Haematol. 2014;167(5):664-70.

53. Molenaar RJ, Botman D, Smits MA, Hira VV, van Lith SA, Stap J, et al. Radioprotection of IDH1-mutated cancer cells by the IDH1-mutant inhibitor AGI-5198. Cancer Res. 2015;75(22):4790-802.

54. Rohle D, Popovici-Muller J, Palaskas N, Turcan S, Grommes C, Campos C, et al. An inhibitor of mutant IDH1 delays growth and promotes differentiation of glioma cells. Science. 2013;340(6132):626-30.

55. Wang P, Wu J, Ma S, Zhang L, Yao J, Hoadley KA, et al. Oncometabolite D-2-hydroxyglutarate inhibits ALKBH DNA repair enzymes and sensitizes IDH mutant cells to alkylating agents. Cell Rep. 2015;13(11):2353-61.

56. Esposito MT, Zhao L, Fung TK, Rane JK, Wilson A, Martin N, et al. Synthetic lethal targeting of oncogenic transcription factors in acute leukemia by PARP inhibitors. Nat Med. 2015;21(12):1481-90. 